\title{
Nonlinear effects in the dynamics of clouds of bubbles
}

\author{
Sanjay Kumar and ChristopherE. Brennen \\ California Institute of Technology, Pasadena, California 91125
}

(Received 1 February 1990; accepted for publication 27 August 1990)

\begin{abstract}
This paper presents a spectral analysis of the response of a fluid containing bubbles to the motions of a wall oscillating normal to itself. First, a Fourier analysis of the Rayleigh-Plesset equation is used to obtain an approximate solution for the nonlinear effects in the oscillation of a single bubble in an infinite fluid. This is used in the approximate solution of the oscillating wall problem, and the resulting expressions are evaluated numerically in order to examine the nonlinear effects. Harmonic generation results from the nonlinearity. It is observed that the bubble natural frequency remains the dominant natural frequency in the volume oscillations of the bubbles near the wall. On the other hand, the pressure perturbations near the wall are dominated by the first and second harmonics present at twice the natural frequency while the pressure perturbation at the natural frequency of the bubble is inhibited. The response at the forcing frequency and its harmonics is explored along with the variation with amplitude of wall oscillation, void fraction, and viscous and surface tension effects. Splitting and cancellation of frequencies of maximum and minimum response due to enhanced nonlinear effects are also observed.
\end{abstract}

PACS numbers: 43.30.Nb, 43.25.Yw

\section{LIST OF SYMBOLS}

$i \quad$ imaginary number

$k \quad$ polytropic constant for gas expansion and contraction

$j, m, n$ index integer

$p \quad$ pressure in liquid flow field

$P_{g 0} \quad$ pressure of permanent gas in the bubble at undisturbed condition

$P_{n} \quad$ complex amplitude of pressure oscillation at frequency $n \delta$

$P_{0} \quad$ reference pressure in the liquid

$P_{v} \quad$ vapor pressure inside the bubble

$P_{\infty} \quad$ pressure at infinity

$R \quad$ radius of the bubble

$R_{0} \quad$ radius of the bubble in reference condition

$R_{n} \quad$ complex amplitude of radius oscillation at frequency $n \delta$

$S \quad$ surface tension of the liquid

$t$ time

$T \quad$ Lagrangian time

\section{INTRODUCTION}

The objective of this work is to gain an understanding of the interactions between individual bubbles in bubbly flows. The dynamics of bubbles can be quite nonlinear. In a bubble cloud, these nonlinear bubble dynamics produce nonlinear interactive effects. At the most basic level, this interaction comes about because the response of the bubble to pressure changes results in volume changes which in turn cause accelerating velocity fields that effect the pressure. Our approach is to understand mechanisms in cavitating flows by studying analytically amenable model problems. The present paper attempts to construct a nonlinear analysis of one such model problem. $u \quad$ velocity in the liquid flow field

$x \quad$ Eulerian space coordinate normal to the wall

$X \quad$ Lagrangian space coordinate normal to the wall

$X_{n} \quad$ complex amplitude of wall oscillation at frequency $n \delta$

$\alpha_{0} \quad$ volume fraction of bubbly mixture at reference condition

$\delta \quad$ increment in the frequency

$\gamma \quad$ ratio of specific heats

$v \quad$ kinematic viscosity

$\omega_{b} \quad$ natural frequency of the bubble ( $\left.\mathrm{rad} / \mathrm{s}\right)$

$\omega_{f} \quad$ forcing frequency for pressure or wall oscillation $(\mathrm{rad} / \mathrm{s})$

$\Re \quad$ real part of complex quantity

$\tau \quad$ volume of the bubble

$\tau_{n} \quad$ complex amplitude of the bubble volume oscillation at frequency $n \delta$

$\tau_{0} \quad$ volume of the bubble at undisturbed condition

$\rho \quad$ density of the liquid

$\rho_{v} \quad$ density of the vapor in the bubble

First, we provide a brief review of previous research in simulating dynamics of bubble clouds and place our work in perspective. The nonlinear dynamics in the growth and collapse of a single bubble have been studied for a long time [e.g., Plesset and Prosperetti (1977)]. Among the first to focus on the dynamics of bubble clusters was van Wijngaarden (1964) who analyzed the collapse of a large number of bubbles next to a flat wall and found considerable increase in the pressure at the wall as a result of the interactive effects. Morch $(1980,1982)$ considered the collapse of a spherical bubble cloud characterized by a cloud radius and uniform volume fraction. He assumed that the pressure increase would lead to shock formation at the cloud boundary and 
that the shock would propagate inward completely annihilating bubbles in its path. This model did not include individual bubble dynamics and predicted infinite pressure and infinite collapse velocities as the radius approached zero. In a subsequent paper, Hansson et al. (1982) constructed a model using a continuum mechanics approach and used the Rayleigh-Plesset equation to model the bubble dynamics. In particular, the response of a bubble cloud to a vibrating horn and the cavitating flow in an accelerating water column were considered. Chahine (1982) developed a method using matched asymptotic expansions. This model assumes instantaneous transmission of ambient conditions to the bubbles and thus neglects the compressibility of the bubble cloud. This is a major weakness in the model because compressibility of the clould will not be negligible for moderate to high void fractions. It was found that because of interactive effects in the cloud, the larger the number of bubbles in the cloud, the more delayed and violent is the implosion and thus the higher are the pressures generated. Chahine (1982) also developed a model using a continuum mechanics approach and first-order gradient theory.

Recently Omta (1987) has carried out analytical solutions for small amplitude oscillations and numerical solutions for large amplitudes. The interior of the bubble was considered nonuniform. Frequency spectra for the bubble cloud were obtained. The natural frequencies of the cloud were found to depend upon void fraction and not upon the bubble size, a feature that seems particularly true at the lowest cloud natural frequency. d'Agostino et al. (1988) solved for the linearized dynamics of the flow of bubbly mixture over slender surfaces. d'Agostino and Brennen (1988) calculated natural frequencies of the bubble cloud and solved the linearized dynamics of spherical bubble clouds. Other than Omta's work very little has been done on the nonlinear solutions of the dynamics of bubble clouds. The objective of present work is to develop a methodology for handling nonlinear terms and to obtain nonlinear solutions by studying the dynamics of a bubbly liquid next to a flat wall that oscillates normal to its own plane.

\section{NONLINEAR SOLUTION OF THE RAYLEIGH- PLESSET EQUATION}

There exists a substantial body of literature on the nonlinear dynamics of a single bubble in an infinite fluid; this has been reviewed by Plesset and Prosperetti (1977). In the present context, it is appropriate to note that Eller and Flynn (1969) solved the problem of subharmonics of order onehalf using a perturbation procedure and that Prosperetti (1974) generated nonlinear analytical solutions for subharmonics and harmonics of various orders using perturbation method.

In the present work, it is necessary to construct the very simplest nonlinear solution of the Rayleigh-Plesset equation for a single bubble. Later, this will be used as a building block for the problem of many bubbles interacting in a flow. The bubble is assumed to be spherical and to contain water vapor and residual permanent gas. The bubble interior is assumed to be uniform with constant vapor pressure, $P_{v}$. The permanent gas in the bubble is assumed to behave polytropically with an index $k$ between 1 and $\gamma$ (Plesset and Hsieh, 1960). The liquid compressibility is only included in the radiation damping, and this is done by including it in the effective viscosity used for the bubble dynamics (Devin, Plesset and Prosperetti, 1959; 1977). With these assumptions, the Rayleigh-Plesset equation describing the bubble dynamics becomes

$$
\begin{gathered}
R \frac{D^{2} R}{D t^{2}}+\frac{3}{2}\left(\frac{D R}{D t}\right)^{2}+\frac{4 v}{R} \frac{D R}{D t}+\frac{2 S}{\rho R} \\
=\frac{P_{v}-P_{\infty}(t)}{\rho}+\frac{P_{g 0}}{\rho}\left(\frac{R_{0}}{R}\right)^{3 k} .
\end{gathered}
$$

In the present solution, a Fourier series expansion is used and terms up to second order are retained in order to examine these corrections to the nonlinear solution. The bubble radius $R(t)$ and the pressure at infinity $P_{\infty}(t)$ are expanded in the form,

$$
\begin{aligned}
& R=R_{0}+\sum_{n=1}^{N} \Re\left(R_{n} e^{i n \delta t}\right), \\
& \frac{P_{\infty}(t)}{\rho}=P_{0}+\sum_{n=1}^{N} \Re\left(P_{n} e^{i n \delta t}\right),
\end{aligned}
$$

where $P_{n}$ and $R_{n}$ are complex quantities and the frequencies $n \delta, n=1$ to $N$, represent a discretization of the frequency domain. These expansions are substituted into Eq. (1) and all terms of third or higher order in $R_{n} / R_{0}$ are neglected in order to extract the simplest nonlinear effects. Finally, coefficients of $e^{i n \delta t}$ on both sides of the simplified equation are equated to yield the following relation for $P_{n}$ and $R_{n}$ :

$$
\begin{aligned}
\frac{P_{n}}{\omega_{b}^{2} R_{0}^{2}}= & \Lambda \frac{R_{n}}{R_{0}}+\sum_{j=1}^{n-1} \beta_{1}(n, j) \frac{R_{j}}{R_{0}} \frac{R_{n-j}}{R_{0}} \\
& +\sum_{j=1}^{N-n} \beta_{2}(n, j) \frac{\bar{R}_{j}}{R_{0}} \frac{R_{n+j}}{R_{0}},
\end{aligned}
$$

where the overbar denotes complex conjugate and the bubble natural frequency $\omega_{b}$ is given by

$$
\omega_{b}=\left(3 k P_{g 0} / \rho R_{0}^{2}-2 S / \rho R_{0}^{3}\right)^{1 / 2},
$$

and $\Lambda, \beta_{1}(n, j)$, and $\beta_{2}(n, j)$ are defined as

$$
\begin{aligned}
\Lambda=\left(\frac{n^{2} \delta^{2}}{\omega b^{2}}-1-i \frac{n \delta}{\omega_{b}} \frac{4 v}{\omega_{b} R_{0}^{2}}\right) & \\
\beta_{1}(n, j)= & \frac{3 k+1}{4}+\frac{3 k-1}{2} \frac{S}{\rho \omega_{b}^{2} R_{0}^{3}} \\
& +\frac{1}{2} \frac{\delta^{2}}{\omega_{b}^{2}}(n-j)\left(n+\frac{j}{2}\right) \\
& +i \frac{2 v}{\omega_{b} R_{0}^{2}} \frac{\delta}{\omega_{b}}(n-j),
\end{aligned}
$$

and

$$
\begin{aligned}
\beta_{2}(n, j)= & \frac{3 k+1}{2}+(3 k-1) \frac{S}{\rho \omega_{b}^{2} R_{0}^{3}} \\
& +\frac{1}{2} \frac{\delta^{2}}{\omega_{b}^{2}}\left(n^{2}-n j-j^{2}\right)+i \frac{2 v}{\omega_{b} R_{0}^{2}} \frac{n \delta}{\omega_{b}} .
\end{aligned}
$$

Using a Newton-Raphson scheme, Eq. (4) is solved iteratively for $R_{n} / R_{0}$ given $P_{n}$, the fluid properties and individual 
TABLE I. Fluid and bubble parameters for the examples presented. These data are for water at $20^{\circ} \mathrm{C}$.

\begin{tabular}{cccccc}
\hline \hline Data set & $\begin{array}{c}R_{0} \\
(\mu \mathrm{m})\end{array}$ & $\begin{array}{c}P_{g 0} \\
(\mathrm{~Pa})\end{array}$ & $k$ & $v / \omega_{b} R_{0}^{2}$ & $S / \rho \omega_{b}^{2} R_{0}^{3}$ \\
\hline I & 20 & $\mathbf{1 4 6 8 0}$ & 1 & 0.01 & 0.10 \\
II & 20 & 106326 & 1 & 0.0028 & 0.0118 \\
\hline
\end{tabular}

bubble characteristics. It is clear that if there is a single forcing frequency, $\omega_{f}$, then the only nonzero components of the bubble oscillation, $R_{n}$, will occur at harmonics of forcing frequency. It is also seen that the response $R_{n} / R_{0}$ decays with increase in the order of the harmonic and is negligible (amplitude $\ll 10^{-20}$ ) at harmonics of order higher than 50 . Thus calculating the response up to 50 harmonics was considered sufficient. It is also clear from the Eqs. (7) and (8) that $\beta_{1}(n, j)$ and $\beta_{2}(n, j)$ are functions of $n \delta / \omega_{b}$ and $j / n$. Furthermore, note from Eq. (4) that for a single forcing frequency, the only coefficients $\beta_{1}(n, j)$ and $\beta_{2}(n, j)$ that enter the calculations are those for which $j$ and $n$ take values corresponding to harmonics of the forcing frequency. Consequently, the only values of $n \delta / \omega_{b}$ and $j / n$ that enter the calculations are those which are ratios between a forcing frequency harmonic and the natural frequency of the bubble or two forcing frequency harmonics. Hence despite the explicit appearance of $\delta$, the results of the calculation are independent of this parameter used in descritizing the frequency domain. Finally, note also that the pressure perturbations, $P_{n}$, occur in (4) only in linear form and thus can be large without introducing error into the solution. However, the analysis is valid only for $\left|R_{n}\right| R_{0} \mid \ll 1$. This defines the extent of the weak nonlinear effects that are examined here and indirectly, implies an upper limit on the magnitude of $P_{n} /$ $\omega_{b}^{2} R_{0}^{2}$.

For illustrative purposes, we select the values of the parameters $v / \omega_{b} R_{0}^{2}$ and $S / \rho \omega_{b}^{2} R_{0}^{3}$ listed as data set I in Table I. The values are for typical cavitating conditions in the ocean or in a water tunnel. We chose to consider a single bubble subjected to an oscillating pressure at infinity containing a single frequency, $\omega_{f}$ with an amplitude $\left|P_{n}\right|$ $\omega_{b}^{2} R_{0}^{2}$. First of all, results obtained from Eq. (4) are compared to a numerical integration of the Rayleigh-Plesset equation that uses a fourth order Runge-Kutta scheme. In Figs. 1 and 2, the radius-time behavior obtained from our analysis and the numerical integration of the Rayleigh-Plesset equation are compared. The ratio $\omega_{b} / \omega_{f}$ is 3 and values of $P_{n} / \omega_{b}^{2} R_{0}^{2}$ for Figs. 1 and 2 are 0.04 and 0.08 , respectively. It can be seen that present approximate analysis works very well for weak nonlinear effects or small values of $P_{n} / \omega_{b}^{2} R_{0}^{2}$. The agreement between the numerical integration and the present solution is less satisfactory for higher values of $P_{n} /$ $\omega_{b}^{2} R_{0}^{2}$, as shown in Fig. 2. A comparison of the spectra of $\left[1-R(t) / R_{0}\right]$ is made in Fig. 3 for the case in which the $P_{n} / \omega_{b}^{2} / R_{o}^{2}$ and $\omega_{b} / \omega_{f}$ values are 0.08 and 6 , respectively. It can be seen that the present approximate solution agrees well with the numerical integration for frequencies at which the magnitude is significant. Note that the radius oscillations

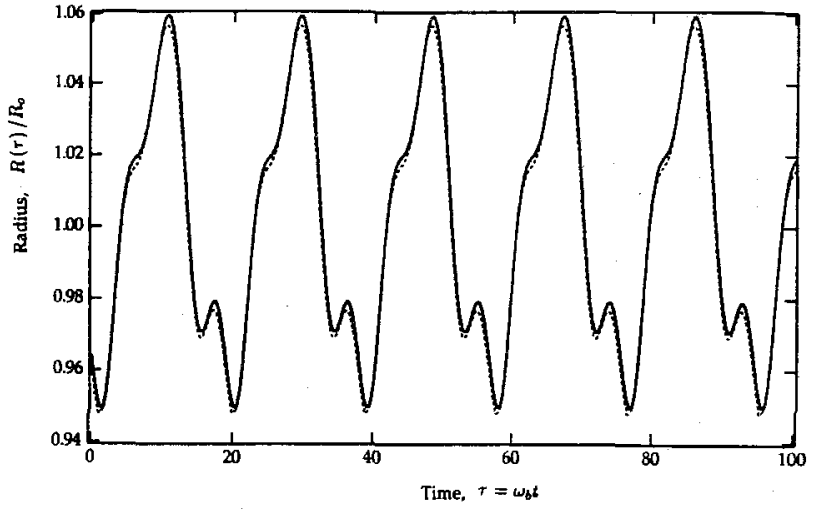

FIG. 1. Radius, $R(\tau) / R_{0}$ plotted against the dimensionless time, $\tau=\omega_{b} t$ for a single bubble. The parameters $P_{n} / \omega_{b}^{2} R_{0}^{2}=0.04, \omega_{b} / \omega_{p}=3.0$ and $v / \omega_{b} R_{0}^{2}$ and $S / \rho \omega_{b}^{2} R_{0}^{3}$ are as in data set I. (- - is the numerical solution and (-.) is the approximate analytical solution.

occur at harmonics of the frequency of the pressure oscillation, $\omega_{f}$. Figure 4 shows the frequency response of a single bubble subjected to a pressure oscillation with $P_{n} / \omega_{b}^{2} R_{0}^{2}$ value of 0.02 . For this purpose, the value of forcing frequency, $\omega_{f}$ is varied from $\omega_{b} / 100$ to $2 \omega_{b}$ and the magnitudes of harmonics of different orders are plotted against the reduced frequency, $n \delta / \omega_{b}$. The lines labeled [1] are the magnitudes of the response at the fundamental forcing frequency, $\omega_{f}$ so that, in this case, the abscissa represents $\omega_{f} / \omega_{b}$. The lines labeled [2] represent the magnitudes of the response at twice the forcing frequency; and in this case abscissa, the represents $2 \omega_{f} / \omega_{b}$. And so on for the lines labeled [3]-[5], which represent the response at the third, fourth, and fifth harmonics of the forcing frequency. All the harmonics are plotted against the actual reduced frequency, $\omega / \omega_{b}$, at which they occur. In this figure, we have presented the results for harmonics up to fifth order. In viewing these results, it should be recognized that those harmonics with magnitudes below a certain level are of dubious significance since higher-order nonlinearities could markedly alter those results. It can be seen that $\omega_{b}$ is the dominant frequency in the radius oscillation as would be expected from the linear analysis.

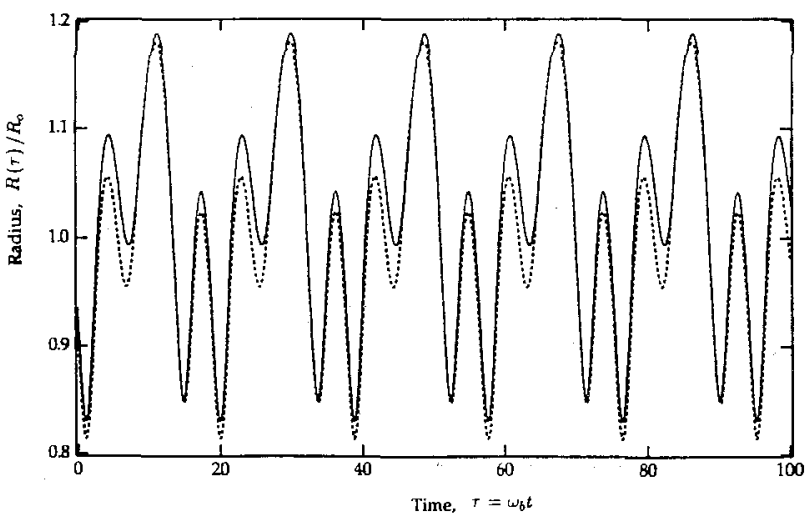

FIG. 2. Radius, $R(\tau) / R_{0}$ plotted against the dimensionless time, $\tau=\omega_{b} t$ for a single bubble. The parameters $P_{n} / \omega_{b}^{2} R_{0}^{2}=0.08, \omega_{b} / \omega_{p}=3.0$ and $v / \omega_{b} R_{0}^{2}$ and $S / \rho \omega_{b}^{2} R_{0}^{3}$ are as in data set $\mathbf{I}$. (- $(-)$ is the numerical solution and (- -) is the approximate analytical solution. 


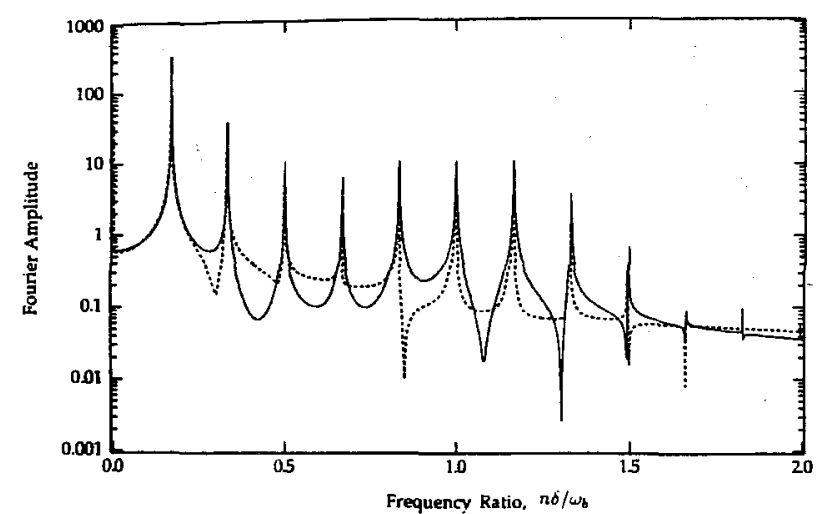

FIG. 3. Comparison of the spectra of $\left[1-R(\tau) / R_{0}\right]$ obtained for a single bubble from numerical integration of the Rayleigh-Plesset equation ( $(-)$ and the present approximate $(-\cdot)$ analysis. The parameters $P_{n} / \omega_{b}^{2} R_{0}^{2}=0.08, \omega_{b} / \omega_{p}=6.0$ and $v / \omega_{b} R_{0}^{2}$ and $S / \rho \omega_{b}^{2} R_{0}^{3}$ are as in data set I.

More accurate nonlinear solutions than the one described above, e.g., Prosperetti (1974), exist and have been reported in the literature. The value of present solution lies in its simplicity and the feasibility of incorporating it in analysis of the collective response of a cloud of bubbles.

\section{NONLINEAR FLAT WALL SOLUTION}

The specific problem addressed in this paper is shown schematically in Fig. 5. Liquid containing bubbles is bounded by a flat wall that oscillates in a direction normal to itself at a given frequency, $\omega_{f}$. The resulting flow is assumed to be function of $x$ and $t$ only. The assumptions behind the continuum model are the same as those described by d'Agostino et al. (1988). The continuity equation is given by

$$
\frac{\partial u}{\partial x}=\frac{\eta}{(1+\eta \tau)} \frac{D \tau}{D t}
$$

where $\eta$ is the population of bubbles per unit liquid volume, a quantity that is both uniform and constant since (a) the relative motion between the bubbles and the liquid is neglected [d'Agostino and Brennen (1989) ], (b) the liquid is assumed incompressible, and (c) the volume of liquid in-

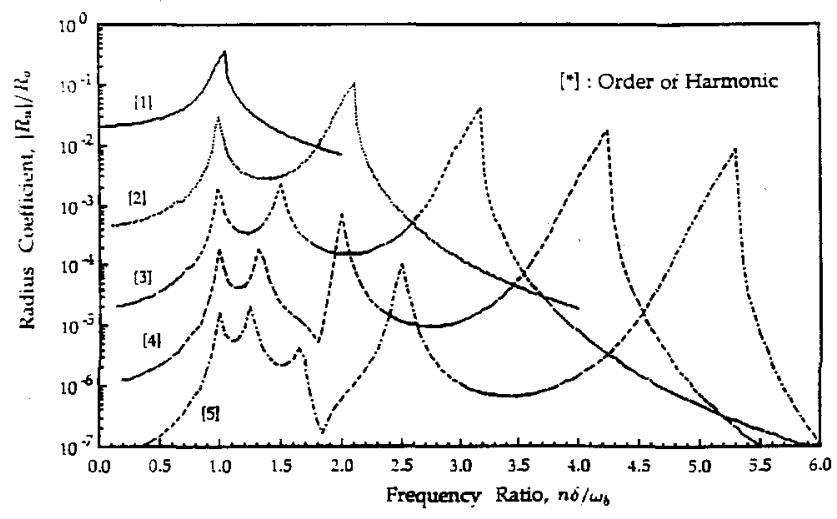

FIG. 4. The frequency response of a single bubble; $\left|R_{n}\right| R_{0}$ is plotted against the frequency ratio, $n \delta / \omega_{b}$ for the first five harmonics. The parameters $P_{n} / \omega_{b}^{2} R_{0}^{2}=0.02$, and $v / \omega_{b} R_{0}^{2}$ and $S / \rho \omega_{b}^{2} R_{0}^{3}$ are as in data set $\mathrm{I}$.

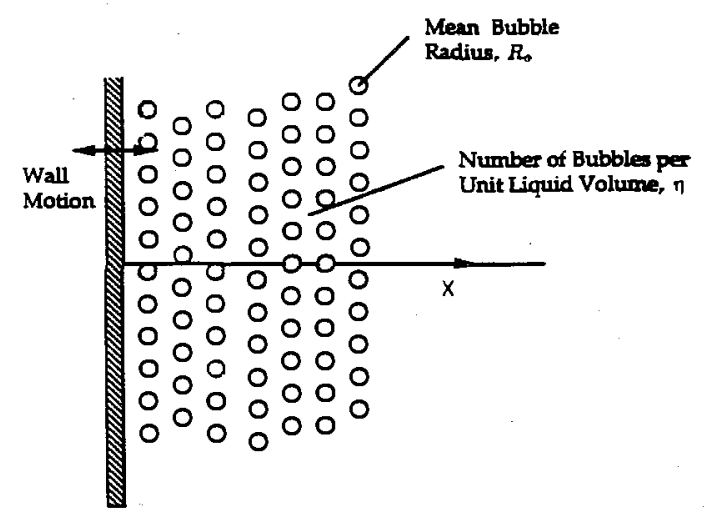

FIG. 5. Schematic of the oscillating wall problem.

volved in condensation or evaporation is neglected. The corresponding momentum equation is

$$
\rho \frac{D u}{D t}=-(1+\eta \tau) \frac{\partial p}{\partial x} .
$$

The solution to the problem represented by Eqs. (9), (10), and (1) is obtained in Lagrangian coordinates, $X$ and $T$ for which the above equations become

$$
(1+\eta \tau) \frac{\partial u}{\partial X}=\eta \frac{\partial \tau}{\partial T} \frac{\partial x}{\partial X}
$$

and

$$
\frac{\partial u}{\partial T} \frac{\partial x}{\partial X}=-(1+\eta \tau) \frac{1}{\rho} \frac{\partial p}{\partial X}
$$

Consistent with the structure of the solution sought, the relationship between the Lagrangian and the Eulerian coordinates, $X$ and $x$, is written in the form,

$$
x=X+\sum_{n=1}^{N} \Re\left[X_{n}(X) e^{i n \delta T}\right],
$$

and the bubble volume, $\tau$, and pressure, $P$, are expressed by the expansions,

$$
\tau=\tau_{0}+\sum_{n=1}^{N} \Re\left[\tau_{n}(X) e^{i n \delta T}\right],
$$

and

$$
\frac{P}{\rho}=P_{0}+\sum_{n=1}^{N} \Re\left(P_{n}(X) e^{i n \delta T}\right) .
$$

The expansions (13)-(15) are substituted into Eq. (11), and coefficients of $e^{i n \delta T}$ are equated to obtain

$$
\begin{aligned}
\frac{d X_{n}}{d X}= & \alpha_{0} \frac{\tau_{n}}{\tau_{0}}+\alpha_{0} \sum_{j=1}^{n-1} \frac{(2 j-n)}{2 n} \frac{\tau_{j}}{\tau_{0}} \frac{d X_{n-j}}{d X} \\
& +\alpha_{0} \sum_{j=1}^{N-n} \frac{(n+2 j)}{2 n} \\
& \times\left(\frac{\tau_{n+j}}{\tau_{0}} \frac{d \bar{X}_{j}}{d X}-\frac{\bar{\tau}_{j}}{\tau_{0}} \frac{d X_{n+j}}{d X}\right) .
\end{aligned}
$$

Similar substitution into the momentum equation [Eq. (12)] leads to 


$$
\begin{aligned}
\frac{d P_{n}}{d X}= & \left(1-\alpha_{0}\right) n^{2} \delta^{2} X_{n} \\
& +\frac{1}{2} \sum_{j=1}^{n-1}\left(\left(1-\alpha_{0}\right)(n-j)^{2} \delta^{2} X_{n-j} \frac{d X_{j}}{d X}\right. \\
& \left.-\alpha_{0} \frac{\tau_{j}}{\tau_{0}} \frac{d P_{n-j}}{d X}\right) \\
+ & \frac{1}{2} \sum_{j=1}^{N-n}\left[\begin{array}{c}
(n+j)^{2} \delta^{2}\left(1-\alpha_{0}\right) X_{j+n} \frac{d X_{j}}{d X} \\
+j^{2} \delta^{2}\left(1-\alpha_{0}\right) \overline{X_{j}} \frac{d X_{j+n}}{d X} \\
-\alpha_{0} \frac{\overline{\tau_{j}}}{\tau_{0}} \frac{d P_{n+j}}{d X}-\alpha_{0} \frac{\tau_{j+n}}{\tau_{0}} \frac{\overline{d P_{j}}}{d X}
\end{array}\right] .
\end{aligned}
$$

Note that in the linear approximation, Eqs. (16) and (17) become

$$
\frac{d X_{n}}{d X}=\alpha_{0} \frac{\tau_{n}}{\tau_{0}}
$$

and

$$
\frac{d P_{n}}{d X}=\left(1-\alpha_{0}\right) n^{2} \delta^{2} X_{n}
$$

It is consistent with the level of approximation to substitute these first-order expressions into the quadratic terms in Eqs. (16) and (17), which then become

$$
\frac{d X_{n}}{d X}=\alpha_{0} \frac{\tau_{n}}{\tau_{0}}+\alpha_{0}^{2} \sum_{j=1}^{n-1} \frac{(2 j-n)}{2 n} \frac{\tau_{j}}{\tau_{0}} \frac{\tau_{n-j}}{\tau_{0}}+O\left(\alpha_{0}^{3}\right)
$$

and

$$
\frac{d P_{n}}{d X}=\left(1-\alpha_{0}\right) n^{2} \delta^{2} X_{n}+O\left(\alpha_{0}^{3}\right)
$$

The simple algebraic relation between the bubble radius and the bubble volume leads to

$$
\frac{\tau_{n}}{\tau_{0}}=3 \frac{R_{n}}{R}+\frac{3}{2} \sum_{j=1}^{n-1} \frac{R_{j}}{R_{0}} \frac{R_{n-j}}{R_{0}}+3 \sum_{j=1}^{N-n} \frac{\overline{R_{j}}}{R_{0}} \frac{R_{n+j}}{R_{0}},
$$

and using this in (20) and (21), one obtains the following equation:

$$
\frac{d^{2}\left(P_{n} / \omega_{b}^{2} R_{0}^{2}\right)}{d\left(x / R_{0}\right)^{2}}=3 \alpha_{0}\left(1-\alpha_{0}\right)\left(\frac{n \delta}{\omega_{b}}\right)^{2} \frac{R_{n}}{R_{0}}+f_{n 1}(X),
$$

where $f_{n 1}(X)$ is given by

$$
\begin{aligned}
f_{n 1}(X)= & 3 \alpha_{0}\left(1-\alpha_{0}\right)\left(n \delta / \omega_{b}\right)^{2} \\
& \times\left[\begin{array}{l}
\sum_{j=1}^{n-1}\left(\frac{1}{2}+\frac{3 \alpha_{0}(2 j-n)}{2 n}\right) \frac{R_{j}}{R_{0}} \frac{R_{n-j}}{R_{0}} \\
+\sum_{j=1}^{N-n} \frac{\overline{R_{j}}}{R_{0}} \frac{R_{n+j}}{R_{0}}
\end{array}\right] .
\end{aligned}
$$

At this point in the solution, we have obtained one relation, Eq. (23), connecting the pressure coefficients, $P_{n}$ to the radius coefficients, $R_{n}$. We now introduce the RayleighPlesset equation that will provide a second such relation. More specifically, we use Eq. (4), which may be written as

$$
\left(P_{n} / \omega_{b}^{2} R_{0}^{2}\right)=\Lambda\left(R_{n} / R_{0}\right)+f_{n 2}(X),
$$

where

$$
\begin{aligned}
f_{n 2}(X)= & \sum_{j=1}^{n-1} \beta_{1}(n, j) \frac{R_{j}}{R_{0}} \frac{R_{n-j}}{R_{0}} \\
& +\sum_{j=1}^{N-n} \beta_{2}(n, j) \frac{\overline{R_{j}}}{R_{0}} \frac{R_{n+j}}{R_{0}},
\end{aligned}
$$

where $\beta_{1}(n, j)$ and $\beta_{2}(n, j)$ are given by Eqs. (7) and (8). For convenience, we define, $\lambda_{n}$, such that

$$
\lambda_{n}^{2}=3 \alpha_{0}\left(1-\alpha_{0}\right)\left(n \delta / \omega_{b}\right)^{2} / \Lambda
$$

Now the linear terms involving the radius coefficients, $R_{n} /$ $R_{0}$, can be eliminated from the simultaneous Eqs. (23) and (25) to yield the following differential equation for the pressure coefficients $P_{n}$ :

$$
\frac{d^{2}\left(P_{n} / \omega_{b}^{2} R_{0}^{2}\right)}{d\left(X / R_{0}\right)^{2}}=\lambda_{n}^{2}\left(\frac{P_{n}}{\omega_{b}^{2} R_{0}^{2}}-f_{n 2}(X)\right)+f_{n 1}(X) .
$$

The solution of this equation has the form,

$$
P_{n} / \omega_{b}^{2} R_{0}^{2}=\Lambda S_{n} e^{-\lambda_{n} X / R_{n}}+f_{n 3}(X),
$$

where from Eqs. (25) and (29), we have

$$
R_{n} / R_{0}=S_{n} e^{-\lambda_{n} X / R_{n}}+\left[f_{n 3}(X)-f_{n 2}(X)\right] / \Lambda,
$$

and using Eqs. (28) and (29),

$$
\frac{d^{2} f_{n 3}}{d\left(x / R_{0}\right)^{2}}=\lambda_{n}^{2}\left[f_{n 3}(X)-f_{n 2}(X)\right]+f_{n 1}(X) .
$$

Having obtained the form of the solution (30) and noting that the linear component of this solution can be written as

$$
R_{n} / R_{0}=S_{n} e^{-\lambda_{n} X / R_{0},}
$$

we can proceed to evaluate $f_{n 1}(X)$ and $f_{n 2}(X)$ from Eqs. (24) and (26) by noting that it is consistent with the level of approximation to use the expressions (32) in the quadratic terms. Then Eq. (31) can be solved exactly, the solution taking the form

$f_{n 3}(X)=\left[\begin{array}{l}\sum_{j=1}^{n-1} \beta_{3}(n, j) S_{j} S_{n-j} e^{-\left(\lambda_{j}+\lambda_{n-j}\right) X / R_{0}} \\ +\sum_{j=1}^{N-n} \beta_{4}(n, j) \overline{S_{j}} S_{n+j} e^{-\left(\overline{\lambda_{j}}+\lambda_{n+j}\right) X / R_{0}}\end{array}\right]$,

where

$$
\begin{aligned}
\beta_{3}(n, j)= & \frac{\lambda_{n}^{2}}{\left(\lambda_{j}+\lambda_{n-j}\right)^{2}-\lambda_{n}^{2}} \\
& \times\left[\begin{array}{l}
\Lambda\left\{\frac{1}{2}+3 \alpha_{0}[(2 j-n) / 2 n]\right\} \\
-\beta_{1}(n, j)
\end{array}\right]
\end{aligned}
$$

and

$\beta_{4}(n, j)=\left[\lambda_{n}^{2} /\left\{\left(\overline{\lambda_{j}}+\lambda_{n+j}\right)^{2}-\lambda_{n}^{2}\right\}\right]\left[\Lambda-\beta_{2}(n, j)\right]$.

Using Eqs. (19), (29), and (33), we get the following equation: 


$$
\begin{aligned}
-\frac{\lambda_{n}}{3 \alpha_{0}} \frac{X_{n}(0)}{R_{0}}= & S_{n}+\sum_{j=1}^{n-1} \beta_{5}(n, j) S_{j} S_{n-j} \\
& +\sum_{j=1}^{N-n} \beta_{6}(n, j) \overline{S_{j}} S_{n+j}
\end{aligned}
$$

where

$$
\begin{aligned}
\beta_{5}(n, j)= & \frac{\lambda_{n}\left(\lambda_{j}+\lambda_{n-j}\right)}{\left(\lambda_{j}+\lambda_{n-j}\right)^{2}-\lambda_{n}^{2}} \\
& \times\left(\frac{1}{2}+3 \alpha_{0} \frac{(2 j-n)}{2 n}-\frac{\beta_{1}(n, j)}{\Lambda}\right)
\end{aligned}
$$

and

$$
\beta_{6}(n, j)=\frac{\lambda_{n}\left(\overline{\lambda_{j}}+\lambda_{n+j}\right)}{\left(\overline{\lambda_{j}}+\lambda_{n+j}\right)^{2}-\lambda_{n}^{2}}\left(1-\frac{\beta_{2}(n, j)}{\Lambda}\right) .
$$

This completes the solution because for given fluid and bubble properties, the values of $\lambda_{n}$ are known through the definition (27). Then, for given $X_{n}$, the values of $S_{n}$ are given by Eq. (36). Hence, $R_{n} / R_{0}$ is known from Eq. (32). Thus using Eqs. (29) and (33), $P_{n} / \omega_{b}^{2} R_{0}^{2}$ can be calculated.

\section{RESULTS AND DISCUSSION}

For a given wall oscillation amplitude to undisturbed bubble radius ratio, $X_{n}(0) / R_{0}$ and given bubble properties the Eq. (36) can be solved for $S_{n}$ (or $R_{n} / R_{0}$ at the wall) using a Newton-Raphson scheme. Then $P_{n} / \omega_{b}^{2} R_{0}^{2}$ at the wall can be calculated using Eqs. (29) and (33).

Equation (36) is similar in structure to the Eq. (4). Thus for wall motion at a single frequency, $\omega_{f}$, the only nonzero response occurs at the harmonics of $\omega_{f}$. For the same reasons as given earlier in the context of Eq. (4), the solutions to the Eq. (36) are independent of the interval of descritization, $\delta$. Also, both $P_{n} / \omega_{b}^{2} R_{0}^{2}$ and $R_{n} / R_{0}$ appear only at harmonics of the frequency of the wall oscillation, $\omega_{f}$. Thus the software may be written so as to evaluate only the response of nonzero amplitude, i.e., at the harmonics. Calculation of the harmonics up to order 50 was found to be sufficient, harmonics of higher order being negligible. For the purpose of demonstrating the nonlinear effects, we choose to vary the wall oscillation frequency from $\omega_{b} / 100$ to $2 \omega_{b}$ and the resulting magnitudes of the harmonics $P_{n} / \omega_{b}^{2} R_{0}^{2}$ and $R_{n} / R_{0}$ at the wall are plotted as functions of the reduced frequency $n \delta / \omega_{b}$. Data for the two sets of values listed in Table I will be presented. A convenient reference case will consist of data set I plus a void fraction, $\alpha_{0}$, of 0.02 and an amplitude of wall oscillation, $X_{n}(0) / R_{0}$, of 0.03 . Data set II will be used to examine the effect of varying the viscous and surface tension parameters. The effect on the results of varying $\alpha_{0}$ and $X_{n}(0) / R_{0}$ will also be examined.

Results for the reference case are presented in Fig. 6 in exactly the same way as the earlier results were presented in Fig. 4 . We reiterate that data below a certain magnitude will be substantially effected by higher-order nonlinearities. The first point to note is that the response rapidly decays at higher harmonics. For the purpose of discussion of the results a frequency at which the response is a maximum will be called

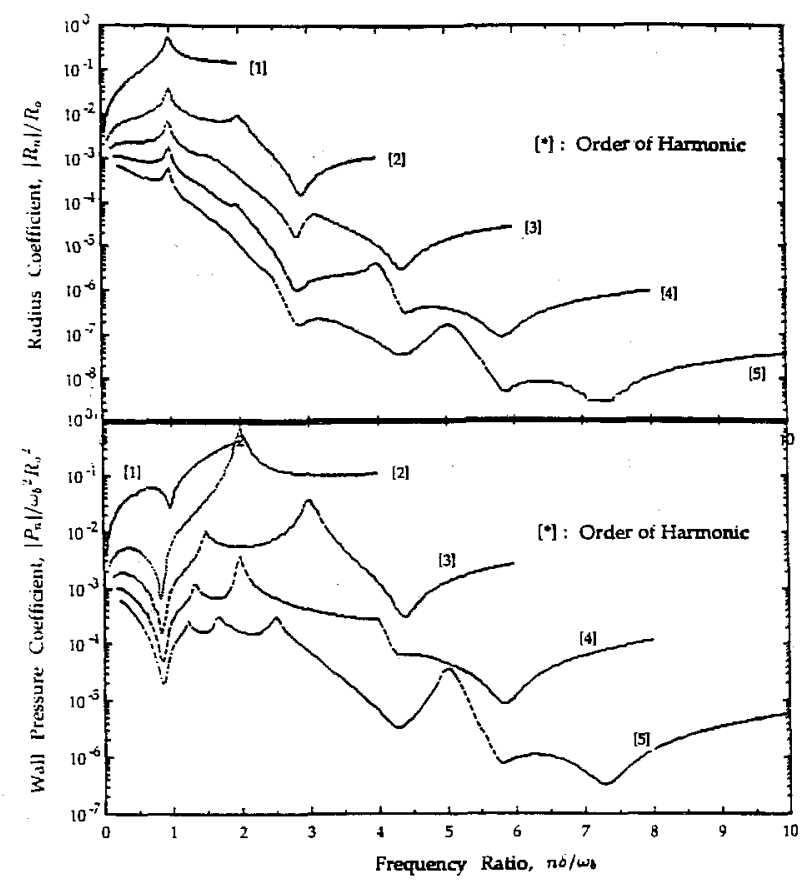

FIG. 6. The frequency response of the bubbly cloud; $\left|R_{n}\right| / R_{0}$ and $\left|P_{n}\right| / \omega_{b}^{2} R_{0}^{2}$ are plotted against the frequency ratio, $n \delta / \omega_{b}$ for the first five harmonics. The parameters $X_{n}(0) / R_{0}=0.03, \alpha_{0}=0.02$ and $v / \omega_{b} R_{0}^{2}$ and $S / \rho \omega_{b}^{2} R_{0}^{3}$ are as in data set $\mathrm{I}$.

an enhancement frequency and a frequency at which the response is a minimum will be called a suppresion frequency. From Fig. 6, it can be seen that the dominant enhancement frequency for bubble radius oscillations at the wall is $\omega_{b}$. Furthemore, harmonics of all orders have a suppression frequency of approximately $3 \omega_{b}$ though the reasons for this are not clear. It can also be seen that the higher harmonics of radius oscillations have other enhancement and suppression frequencies.

In contrast to the radius oscillations, all of the harmonics of the pressure have suppression frequencies close to $\omega_{b}$. The suppression in fundamental harmonic at $\omega_{b}$ is also predicted by the linear solution. It can be seen that the dominant second harmonic occurs at approximately $2 \omega_{b}$ and the dominant third harmonic at approximately $3 \omega_{b}$. The fundamental harmonic in the pressure oscillation reaches a minimum at approximately $\omega_{b}$ and then increases linearly with frequency. Thus the pressure response is dominated by a combination of the fundamental frequency and the second harmonic. In particular, the dominant pressure oscillation occurs at $2 \omega_{b}$. This high-pressure response at the second harmonic is one of the main results that emerges from this nonlinear analysis. It can be visualized as follows. When the wall is oscillated close to $\omega_{b}$, the bubble volume response is sufficiently large to cause significant accelerations in the fluid. Thus high pressure fluctuations can be expected. However these occur primarily at $2 \omega_{b}$.

The effect of changing the viscous and the surface tension parameters while all other parameters remain unchanged is illustrated in Fig. 7. This contains a comparison between the results for the data set II and the earlier results for the data set I. Higher viscous and surface tension parameters tend to inhibit bubble oscillations. Hence, the response 


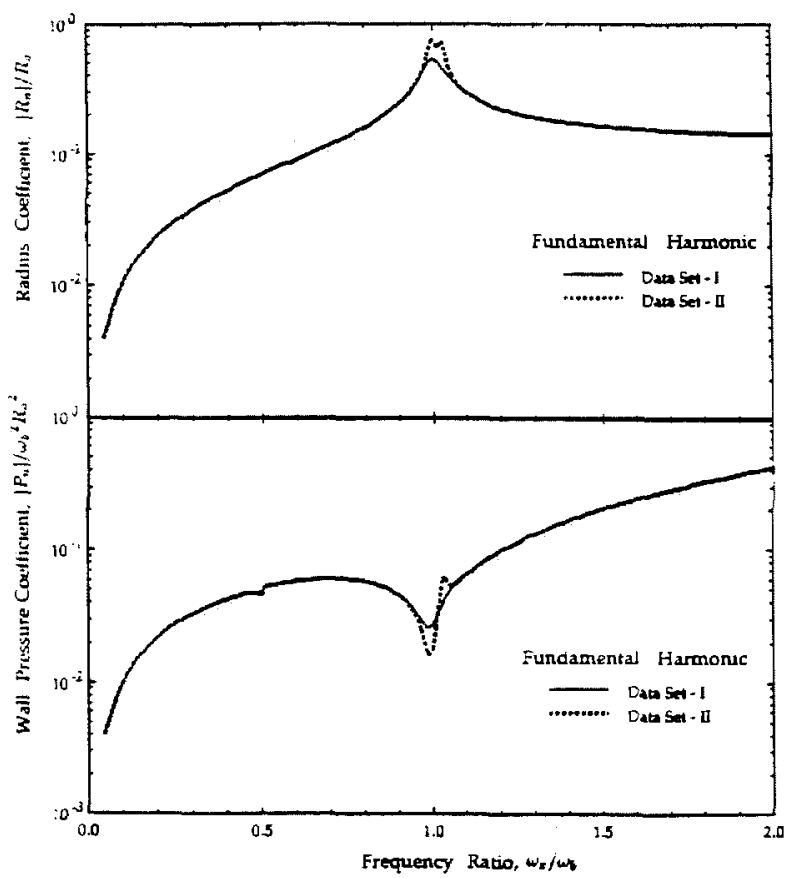

FIG. 7. The effect of variation in $v / \omega_{i} R_{0}^{2}$ and $S / \rho \omega_{i}^{2} R_{i}^{3}$ on the fundamental harmonic; $\left|R_{t i}\right| / R_{t}$ and $\left|\boldsymbol{P}_{t,}\right| / \omega_{b}^{2} \boldsymbol{R}_{0}^{2}$ for the fundamental harmonic are plotted against the frequency ratio, $\omega_{\checkmark} / \omega_{b}$. The parameters $X_{n}(0) /$ $R_{i b}=0.03, \alpha_{0}=0.02$ and $v / \omega_{b}, R_{3}^{2}$ and $S / \rho \omega_{b}^{2} R_{i j}^{2}$ are as in data sets $I$ and II.

curves for the data set Il exhibit sharper peaks and troughs. Otherwise, the basic form of the response is very similar for the two sets of data with only minor differences in enhancement and suppression frequencies due to differences in the levels of nonlinearity. Two additional features of the results presented in Fig. 7 deserve special attention. First, note that the stronger nonlinearity present at the enhancement frequency for the data set II has resulted in splitting into two adjacent enhancement frequencies. This is exemplified in Fig. 7 by the response in the radius oscillation at the fundamental frequency. It also occurs in the second, third, and fifth harmonics of pressure. A different kind of frequency splitting occurs where a suppression frequency splits into a suppression frequency and an enhancement frequency. This can be seen in the fundamental component of pressure oscillation near the frequency ratio of unity.

Next, the effect of varying the void fraction is demonstrated in Fig. 8 where data for void fraction values of 0.005 , 0.020 , and 0.100 are compared. Note that the main features of the results, namely the enhancement and suppression frequencies remain almost the same. However, the nonlinear response is enhanced as the void fraction is reduced. This dependence can be predicted from the linear solution in which both radius and pressure are given by terms multiplied by the factor $\left[\left(1-\alpha_{0}\right) / 3 \alpha_{0}\right]^{1 / 2}$. For the small void fractions considered here, the denominator dominates this factor and implies larger effects at smaller void fractions. Also with the increased level of nonlinearity, the frequency splitting phenomena, described earlier, are observed. Also, the first enhancement frequency in the radius oscillations increases slightly with increased level of nonlinearity. Similarly, the first suppression frequency for pressure oscilla-

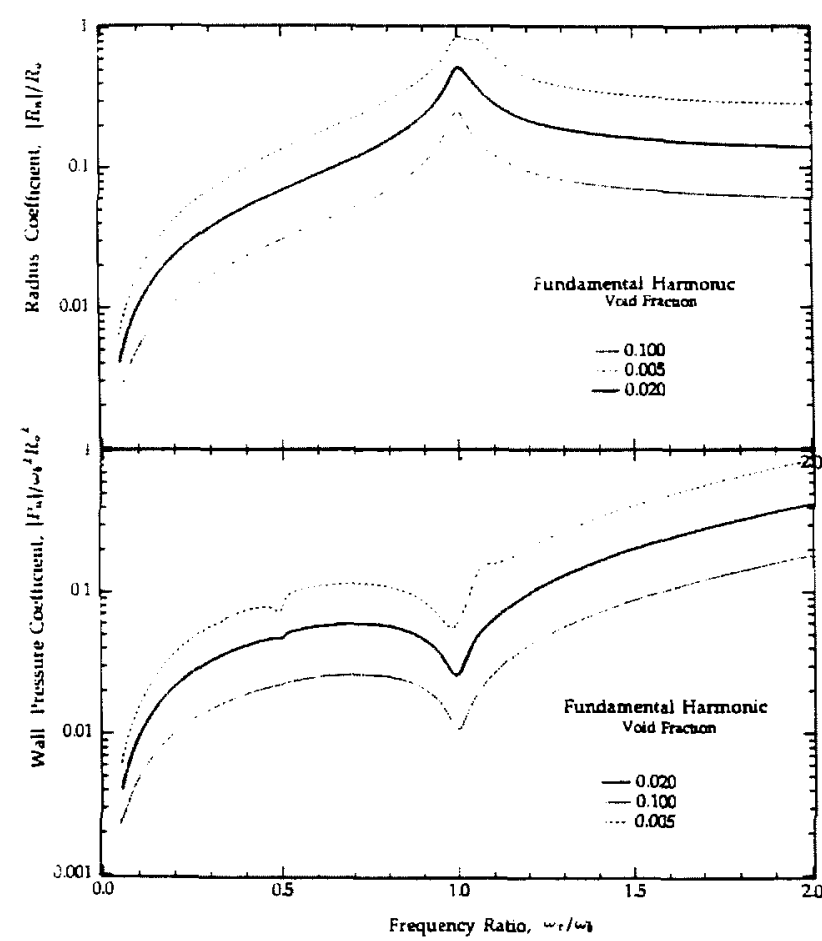

FIG. 8. The effect of change in the void fraction, $\alpha_{1}$ on the fundamental harmonic; $\left|R_{s,}\right| / R_{11}$ and $P_{n} \mid / \omega_{b}^{2} R_{0}^{2}$ for the fundamental harmonic are plotted against the frequency ratio, $\omega_{*} / \omega_{i}$. The parameters $X_{i i}(0) / R_{i}=0.03$ and $v / \omega_{i r} R_{i}^{2}$ and $S / \rho \omega_{i}^{2} R_{i}^{3}$ are as in data set I. Values of the void fraction, $\alpha_{13}$, of $0.005,0.020$, and 0.100 are used.

tions decreases slightly with increased level of nonlinearity. This also occurs in the higher harmonics.

Finally, the effect of changing the amplitude of the wall motion $X_{n z}(0) / R_{0}$ while keeping void fraction constant at

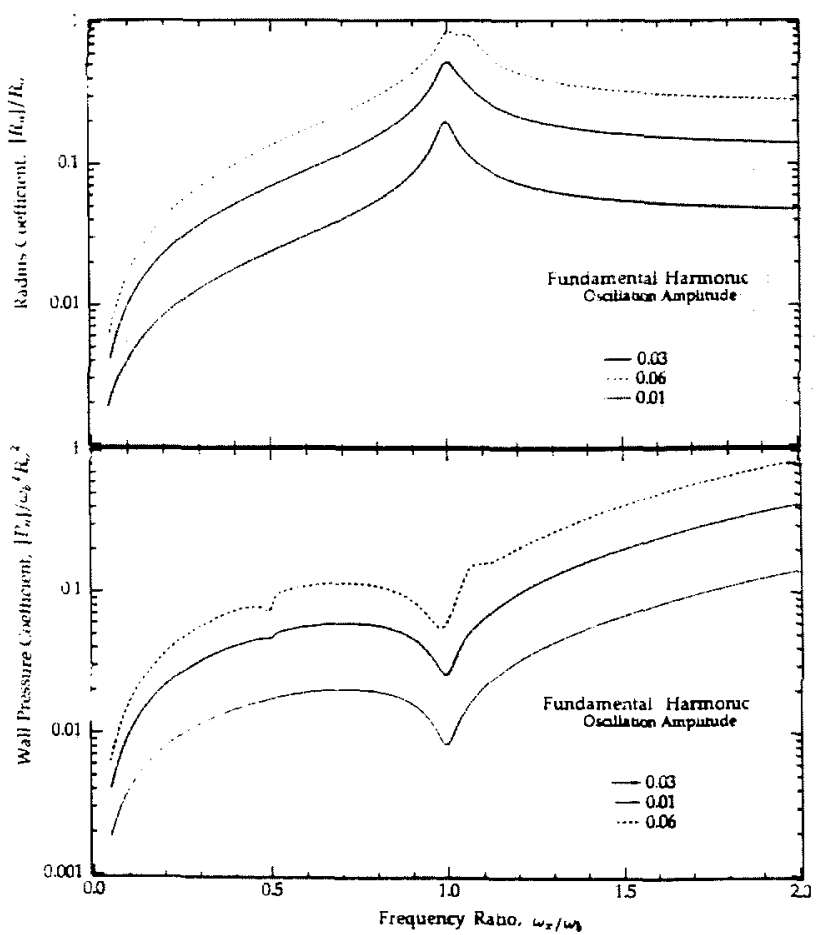

FlG. 9. The effect of change in the amplitude of wall oscillation, $X_{n}(0) / R_{1}$ on the fundamental harmonic; $R_{n, 1} / R_{t}$ and $P_{n} \mid / \omega_{b}^{2} R_{1}^{2}$ for the fundamental harmonic are plotted against the frequency ratio, $\omega_{1} / \omega_{n}$. The parameters $\alpha_{0}=0.02$ and $v / \omega_{i} R_{i}^{2}$ and $S / \rho \omega_{b}^{2} R_{0}^{\vdots}$ values are as in data set $I$. $X_{i}(0) / R_{i}$ values of $0.01,0.03$, and 0.06 are used. 
0.02 is shown in Fig. 9. Values of $0.01,0.03$, and 0.06 for $X_{n}(0) / R_{0}$ are used. Obviously the nonlinear effects become stronger for higher values of $X_{n}(0) / R_{0}$. Splitting of some enhancement and suppression frequencies is observed. In addition, cancellation of neighboring suppression and enhancement frequency can be observed for the fourth harmonic in the radius and pressure oscillations.

\section{CONCLUSIONS}

In this paper, we have examined some of the nonlinear effects that can occur when a plane wall bounding a bubbly liquid oscillates in a direction normal to the plane of the wall. Specifically, we have examined the response in terms of the bubble radius and pressure oscillations at the wall. The principal results are as follows: Radius oscillations are dominated by the fundamental response at the bubble natural frequency. On the other hand, the pressure oscillations at the wall are suppressed near the bubble natural frequency for harmonics of all orders. The pressure oscillations are dominated by the fundamental and the second harmonic responses, primarily at frequencies of approximately $2 \omega_{b}$. It is observed that nonlinear effects increase not only with wall amplitude but also as a result of a decrease in the surface tension and viscous parameters or a decrease in the void fraction. Characteristic suppression and enhancement frequencies depend upon the level of nonlinearity. Also, increased nonlinearity is manifested in the form of splitting and cancellation of enhancement and suppression frequencies.

\section{ACKNOWLEDGMENTS}

The authors are very grateful for the support of the Office of Naval Research under Contract N00014-85-K-0397. We also thank Professor Allan J. Acosta and Professor Sheldon Green for their assistance.

Chahine, G. L. (1982). "Cloud cavitation: Theory," 14th Symp. on Naval Hydrodynamics, August 23-27.

d'Agostino, L., Brennen, C. E., and Acosta, A. J. (1988). "Linearized dynamics of two-dimensional bubbly and cavitating flows over slender surfaces," J. Fluid Mech. 192, 485-509.

d'Agostino, L., and Brennen, C. E. (1988). "Linearized dynamics of spherical bubble clouds," J. Fluid Mech. 199, 155-176.

Devin, C., Jr. (1959). "Survey of thermal, radiation and viscous damping of pulsating air bubbles in water," J. Acoust. Soc. Am. 31, 1654-1667.

Eller, A., and Flynn, H. G. (1969). "Generation of subharmonics of order one-half by bubbles in sound field," J. Acoust. Soc. Am. 46, 722-727.

Hansson, I., Kendrinskii, V., and Morch, K. A. (1982). "On the dynamics of cavity clusters," J. Phys. D 15, 1725-1734.

Morch, K. A. (1980). "On the collapse of cavity clusters in flow cavitation," in Cavitation and Inhomogeneities in Underwater Acoustics, edited by W. Lauterborn (Springer, New York) 4, 95-100.

Morch, K. A. (1982). "Energy considerations in the collapse of cavity clusters," Appl. Sci. Res. 38, 313-321.

Omta, R. (1987). "Oscillations of a cloud of bubbles of small and not so small amplitude," J. Acoust. Soc. Am. 82, 1018-1033.

Plesset, M. S., and Hsieh, D. Y. (1960). "Theory of gas bubble dynamics in oscillating pressure fields," Phys. Fluids 3, 6, 882-892.

Plesset, M. S., and Prosperetti, A. (1977). "Bubble dynamics and cavitation," Ann. Rev. Fluid Mech. 9, 145-186.

Prosperetti, A. (1974). "Viscous and nonlinear effects in the oscillations of drops and bubbles," Ph.D. thesis, California Institute of Technology.

van Wijngaarden, L. (1964). "On the collective collapse of large number of gas bubbles in water," Proceedings of 11 th International Congress of Applied Mechanics, Munich, Germany, pp. 854-861. 